\title{
CB1 cannabinoid receptors mediate cognitive deficits and structural plasticity changes during nicotine withdrawal
}

Rocio Saravia ${ }^{1}$, África Flores ${ }^{1}$, Ainhoa Plaza-Zabala ${ }^{1 \#}$, Arnau Busquets-García ${ }^{1+}$, Antoni Pastor ${ }^{2,5}$, Rafael de la Torre ${ }^{2,5}$, Vincenzo Di Marzo ${ }^{3}$, Giovanni Marsicano ${ }^{4}$, Andrés Ozaita ${ }^{1}$, Rafael Maldonado ${ }^{1}$ and Fernando Berrendero ${ }^{1 *}$

${ }^{1}$ Laboratory of Neuropharmacology, Department of Experimental and Health Sciences, Universitat Pompeu Fabra, PRBB, C/ Doctor Aiguader 88, 08003 Barcelona, Spain

${ }^{2}$ Integrative Pharmacology and Systems Neuroscience, IMIM-Hospital del Mar Medical Research Institute, Barcelona, Spain

${ }^{3}$ Endocannabinoid Research Group, Institute of Biomolecular Chemistry, National Research Council, Via Campi Flegrei 34, Comprensorio Olivetti, 80078 Pozzuoli, Naples, Italy

${ }^{4}$ INSERM, U862 NeuroCentre Magendie, Group Endocannabinoids and Neuroadaptation, Bordeaux 33077, France; University of Bordeaux, Bordeaux 33077, France

${ }^{5}$ CIBER Fisiopatología Obesidad y Nutrición (CIBERObn), Instituto Salud Carlos III, Madrid, Spain

*Corresponding author: Fernando Berrendero, Phone: +34-93-3160890; Fax: + 34-933160901; E-mail: fernando.berrendero@upf.edu;

\# Present address: Achucarro Basque Center for Neuroscience, Bizkaia Science and Technology Park, Zamudio, Spain

+Present address: INSERM, U862 NeuroCentre Magendie, Group Endocannabinoids and Neuroadaptation, Bordeaux 33077, France; University of Bordeaux, Bordeaux 33077, France

Keywords: nicotine, withdrawal, cognition, plasticity, CB1R, knockout mice Short title: Nicotine withdrawal-induced cognitive deficits

Number of words (abstract): 244

Number of words (text): 3969

Number of Figures: 4 Number of Tables: 0

Supplemental Material: Methods, 8 Figures, 1 Table 


\section{Abstract}

Background: Tobacco withdrawal is associated with deficits in cognitive function including attention, working memory and episodic memory. Understanding the neurobiological mechanisms involved in these effects is crucial because cognitive deficits during nicotine withdrawal may predict relapse in humans.

Methods: We investigated the role of CB1 cannabinoid receptors (CB1Rs) in memory impairment and spine density changes induced by nicotine withdrawal precipitated by the nicotinic antagonist mecamylamine. Drugs acting on the endocannabinoid system and genetically modified mice were used.

Results: Memory impairment during nicotine withdrawal was blocked by the CB1R antagonist rimonabant or the genetic deletion of $\mathrm{CB} 1 \mathrm{R}$ in forebrain GABAergic neurons (GABA-CB1R). An increase of 2-arachidonoylglycerol (2-AG), but not anandamide, was observed during nicotine withdrawal. The selective inhibitor of 2-AG biosynthesis O7460 abolished cognitive deficits of nicotine abstinence, while the inhibitor of 2-AG enzymatic degradation JZL184 did not produce any effect in cognitive impairment. Moreover, memory impairment was prevented by the selective mTOR inhibitor temsirolimus and the protein synthesis inhibitor anysomicin. Mature dendritic spines on CA1 pyramidal hippocampal neurons decreased 4 days after the precipitation of nicotine withdrawal, when the cognitive deficits were still present. Indeed, a correlation between memory performance and mature spine density was found. Interestingly, these structural plasticity alterations were normalized in GABA-CB1R conditional knockout mice and after subchronic treatment with rimonabant.

Conclusions: These findings underline the interest of $\mathrm{CB} 1 \mathrm{R}$ as a target to improve cognitive performance during early nicotine withdrawal. Cognitive deficits in early abstinence are associated with increased relapse risk. 


\section{Introduction}

Tobacco consumption is one of the main public health problems worldwide and represents the leading cause of preventable premature mortality in the world. It is estimated that globally, $22.5 \%$ of adults (32\% males, $7 \%$ females) currently smoke tobacco (1). Cessation from tobacco smoking produces numerous undesirable effects, including physical, affective and cognitive symptoms (2). Increasing attention has currently focused on cognitive impairments that emerge during smoking abstinence. Nicotine withdrawal alters a variety of cognitive processes in humans including impairments in attention, working memory and episodic memory $(3,4)$, which can be partially restored by nicotine replacement therapy (4). These cognitive deficits peak within the first few days of tobacco cessation and are gaining importance as a core dependence phenotype and a target for medication development efforts (5). Indeed, cognitive impairment during early nicotine abstinence may promote short-term smoking relapse (6), and pharmacotherapies that increase cognitive performance during nicotine withdrawal may represent potential new drugs for smoking cessation (7). Consistent with this hypothesis, the partial agonist of the $\alpha 4 \beta 2$ nicotinic acetylcholine receptors varenicline, currently used for the treatment of tobacco dependence, enhances mood and cognitive function during early nicotine abstinence (8). These effects seem to be important for the efficacy of varenicline as a smoking cessation agent (8). According to human studies, withdrawal from chronic nicotine results in cognitive deficits in rodent models $(9,10)$, providing an excellent tool to investigate the neural substrates that mediate the effects of nicotine abstinence on cognition.

The endocannabinoid system regulates a range of physiological functions including reward processing, learning and memory $(11,12)$. An important role for the endocannabinoid system in the modulation of the addictive properties of nicotine has 
been clearly established $(13,14)$. Thus, CB1 and CB2 cannabinoid receptor (CB1R and CB2R) antagonism reduces nicotine self-administration in rodents $(15,16)$, while an involvement of $\mathrm{CB} 1 \mathrm{R}$, but not $\mathrm{CB} 2 \mathrm{R}$, has been revealed in the processes driving to relapse to nicotine seeking behavior $(17,18)$. Accordingly, CB1R blockade improved the ability of smokers to quit smoking in randomized clinical trials (19). However, the possible involvement of the endocannabinoid system in the neurobiological substrate underlying the cognitive deficits associated with nicotine withdrawal remains unexplored.

In this study, by using specific pharmacological agents acting on the endocannabinoid system and genetically modified mice, we investigated the role played by CB1R in the cognitive deficits and structural plasticity alterations associated with nicotine withdrawal. 


\section{Methods \& Materials}

\section{Animals}

Experiments were performed in male C57BL/6J mice (Charles River) and in male CB1R constitutive knockout $(\mathrm{KO})$ mice and conditional $\mathrm{KO}$ mice and their wild-type (WT) littermates (8 to 12 weeks old). Conditional $\mathrm{KO}$ mice lacking CB1R in forebrain GABAergic neurons (GABA-CB1R), cortical glutamatergic neurons (Glu-CB1R), in both type of neurons (GABA/Glu-CB1R) and their WT littermates were in a mixed genetic background, with a predominant C57BL/6N contribution (at least 7 generations of backcrossing) (20,21). See Supplement 1 for further details.

\section{Drugs}

(-)-Nicotine hydrogen tartrate salt [(-)-1-methyl-2(3-pyridyl) pyrrolidine] (Sigma) was dissolved in physiological saline $(0.9 \% \mathrm{NaCl})$ and administered at the dose of $25 \mathrm{mg} / \mathrm{kg} /$ day by using subcutaneously (sc) implanted osmotic minipumps. Nicotine dose was calculated as (-)-nicotine hydrogen tartrate salt. Mecamylamine hydrochloride $(2 \mathrm{mg} / \mathrm{kg}$, Sigma) was dissolved in physiological saline and administered sc. The CB1 antagonist rimonabant $(1 \mathrm{mg} / \mathrm{kg}$, Sanofi-Aventis Recherche) and the antagonist of the metabotropic glutamate receptor 5 (mGluR5) MTEP (10mg/kg, Tocris) were diluted in $5 \%$ ethanol, $5 \%$ cremophor, $90 \%$ saline and administered intraperitoneally (ip). The mTOR inhibitor temsirolimus (1mg/kg, ip, LC Laboratories) was dissolved in $2 \%$ ethanol, $8 \%$ cremophor and $90 \%$ saline. The protein synthesis inhibitor anisomycin $(6 \mathrm{mg} / \mathrm{kg}$, ip, Sigma) was dissolved in physiological saline. The diacylglycerol lipase (DAGL) inhibitor O7460 (12mg/kg, ip) was kindly provided by the laboratory of Dr. Vincenzo Di Marzo (Pozzuoli, Italy) (22) and diluted in 10\% DMSO and 90\% saline. The monoacylglycerol lipase (MAGL) inhibitor JZL184 (8 and 20mg/kg, ip, Tocris) 
was dissolved in DMSO. Corticosterone (3mg/kg, ip, Sigma) was dissolved in $10 \%$ ethanol and $90 \%$ saline. All drugs were administered in a volume of $10 \mathrm{ml}$ per $\mathrm{kg}$ of body weight, except for O7460 and JZL184 that were administered in $5 \mathrm{ml} / \mathrm{kg}$ and $2 \mathrm{ml} / \mathrm{kg}$, respectively. Doses of the different drugs used were based on previous studies $(22-25)$.

\section{Nicotine treatment and withdrawal}

Nicotine dependence was induced by using Alzet osmotic minipumps (Model 2002, Alzet®, Cupertino, USA). Minipumps previously filled with saline or nicotine solution were implanted sc in mice under brief isofluorane anesthesia. See Supplement 1 for details.

\section{Object-recognition task}

In preclinical rodent models, some components of episodic memory can be evaluated by an object-recognition task (26). We used this behavioral test to evaluate the presence of cognitive deficits during nicotine withdrawal. See Supplement 1 for details.

\section{Endocannabinoid quantification}

Endocannabinoid levels were measured as previously reported (24), with slights modifications. See details in Supplement 1.

\section{Ballistic labeling with the fluorescent dye DiI}

Four days after the precipitation of nicotine withdrawal, when the cognitive deficits were still present, we evaluated possible changes in dendritic spines density in CA1 
pyramidal neurons of the hippocampus (HPC) and pyramidal medial prefrontal cortex (mPFC) neurons. See Supplement 1 for further details.

\section{Immunoblot analysis}

See Supplement 1

\section{Data analysis}

Data were analyzed using unpaired Student t test, one-way analysis of variance (ANOVA) with treatment as between group factor, or two-way ANOVA with nicotine and pretreatment/genotype as between factors of variation. Subsequent post-hoc analysis (Newman-Keuls) was used when required. The Pearson correlation coefficient was used to analyze the relationship between discrimination index values and mature spine density. Comparisons were considered to be statistically significant when the level of significance was $\mathrm{P}<0.05$. 


\section{Results}

\section{CB1R mediates the cognitive deficits associated with nicotine withdrawal}

The object-recognition test was used to evaluate the presence of cognitive deficits during nicotine withdrawal. Nicotine abstinence was precipitated by mecamylamine injection $(2 \mathrm{mg} / \mathrm{kg}) 20$ minutes after the training phase, and memory was evaluated 24 hours later. Mecamylamine did not produce any effect on memory by itself ( 1 and 2 $\mathrm{mg} / \mathrm{kg}$ ) (Figure S1). Nicotine-treated animals showed lower discrimination index after the precipitation of withdrawal compared with the saline-treated group $(\mathrm{p}<0.01)$ (Figure 1A). To exclude an effect of the chronic nicotine treatment on memory, we performed the object-recognition task after 13 days of minipump implantation. We found no differences between animals chronically treated with nicotine or saline (Figure 1B), thus corroborating that the reduced discrimination index previously observed is only due to the precipitation of nicotine withdrawal. The administration of a low dose of the CB1R antagonist rimonabant $(1 \mathrm{mg} / \mathrm{kg}), 20$ minutes before the precipitation of nicotine withdrawal blocked the memory impairment. Thus, two-way ANOVA showed an interaction between treatment and pretreatment $\left(\mathrm{F}_{1,20}=10.77, \mathrm{p}<0.01\right)$, and post hoc analysis revealed the effect of the CB1R antagonist ( $\mathrm{p}<0.01$ ) (Figure 1C). In agreement, nicotine withdrawal did not induce memory deficits in CB1R KO mice $(p<0.05)$ (interaction treatment $x$ genotype: $F_{1,17}=5.74, p<0.05$ ) (Figure 1D). To further elucidate the neuronal type expressing $\mathrm{CB} 1 \mathrm{R}$ responsible for this effect, we used CB1R conditional KO mice lacking CB1R primary from cortical glutamatergic neurons (GluCB1R) or forebrain GABAergic neurons (GABA-CB1R) (20,21). Interestingly, memory impairment was prevented in GABA-CB1R KO mice $(\mathrm{p}<0.01)$ and in double GABA/Glu CB1R KO mice $(\mathrm{p}<0.01)$ (treatment effect: $\mathrm{F}_{1,47}=6.41, \mathrm{p}<0.05$; genotype effect: $\left.F_{3,47}=3.15, p<0.05\right)$. In contrast, memory deficits were still present in Glu-CB1R 
$\mathrm{KO}$ mice (Figure 1E). Total exploration time was not altered in any of the different experimental groups, except a reduction observed in the case of Glu-CB1R KO animals (Figure S2), as previously reported $(27,28)$. Basal anxiety-like behavior is not modified in these mutant mice (29), and it is suggested that the presence of a stressful stimulus (i.e. new object) is required to allow observable phenotype differences between GluCB1R KO and WT mice (29). All together, these data suggest that activation of CB1R in GABAergic neurons is necessary to reveal memory deficits during nicotine withdrawal. Nevertheless, we cannot rule out the participation of other subpopulations of $\mathrm{CB} 1 \mathrm{R}$ in these cognitive defects since drugs in the pharmacological experiments were peripherally injected.

\section{2-arachidonoylglycerol is involved in the cognitive deficits associated with nicotine}

\section{withdrawal}

We next evaluated the possible endocannabinoid responsible for the memory deficits of nicotine abstinence. A liquid chromatography-mass spectrometry analysis revealed that 2-arachidonoylglycerol (2-AG), but not anandamide (AEA), levels increased in whole brain homogenates extracted 10 minutes after the precipitation of nicotine withdrawal $(\mathrm{p}<0.05)$ (Figure 2A-C). Protein expression levels of MAGL were reduced at this time point in nicotine abstinent animals under our experimental conditions (Figure 2D), while DAGL- $\alpha$ protein levels were not modified. These results suggest that a decrease of degradation could explain the observed enhancement of 2-AG. This endocannabinoid only exhibited a tendency to increase 30 minutes after the precipitation of this syndrome (Figure S3). Activation of mGluR5 can induce the synthesis and release of endocannabinoids, mainly 2-AG (30). Administration of the mGluR5 antagonist MTEP $(10 \mathrm{mg} / \mathrm{kg})$ reversed the cognitive deficits induced by nicotine abstinence, as revealed by 
two-way ANOVA (interaction treatment x pretreatment: $\mathrm{F}_{1,25}=13.80, \mathrm{p}<0.01$ ) and post hoc analysis $(\mathrm{p}<0.01)$ (Figure 2E). 2-AG is synthesized by DAG lipases, while is mainly degraded by the hydrolytic enzyme MAGL (31). Interestingly, the administration of the specific DAGL inhibitor O7460 (12mg/kg) (22) abolished the memory impairment associated with nicotine abstinence $(\mathrm{p}<0.01)$, as shown by twoway ANOVA (interaction treatment $x$ pretreatment: $F_{1,33}=13.15, p<0.01$ ) (Figure $2 F$ ). In contrast, the injection of the MAGL inhibitor JZL184 (8 and 20mg/kg) did not significantly alter these cognitive deficits (interaction treatment $\mathrm{x}$ pretreatment: $\mathrm{F}_{2,45}=2.71$, NS) (Figure 2G). Indeed, JZL184 treatment in control mice tended to decrease the discrimination index at the dose of $20 \mathrm{mg} / \mathrm{kg}$ (Figure $2 \mathrm{G}$ ), suggesting that the enhancement of 2-AG levels can impair learning and memory performance, as recently reported (32). Taken together, these results point to a relevant role for 2-AG in the appearance of cognitive deficits during nicotine withdrawal.

Dysregulation of mTOR signaling has been related to neurodevelopmental and neuropsychiatric disorders, including those related to intellectual disability (33). Moreover, amnesic effects of $\Delta^{9}$-tetrahydrocannabinol (THC), the main psychoactive constituent of Cannabis sativa, are mediated through the activation of this pathway (23). Notably, the mTOR pathway inhibitor temsirolimus $(1 \mathrm{mg} / \mathrm{kg})$ blocked the cognitive deficits associated with nicotine withdrawal, as shown by two-way ANOVA (interaction treatment $\mathrm{x}$ pretreatment: $\mathrm{F}_{1,28}=19.07, \mathrm{p}<0.01$ ), and post hoc comparisons $(\mathrm{p}<0.01)$ (Figure 2H). mTOR signaling affects memory processes through its involvement in protein synthesis (34). Although it has been established that long-term memory formation requires the synthesis of new proteins, an aberrant increase in protein synthesis can also lead to memory impairment $(23,35)$. Interestingly, the injection of a non-amnesic dose of the protein synthesis inhibitor anisomycin $(6 \mathrm{mg} / \mathrm{kg})$ 
(Figure 2I) rescued memory impairment $(\mathrm{p}<0.01)$ of nicotine abstinence, as revealed by two-way ANOVA (interaction treatment x pretreatment: $F_{1,25}=8.79, p<0.01$ ) (Figure $2 J$ ). Total exploration time was not modified in any of the different experiments performed (Figure S4). These results suggest that activation of the mTOR pathway and an excess of protein synthesis could negatively affect cognition during nicotine withdrawal.

\section{Structural plasticity alterations during nicotine withdrawal are normalized by CB1R blockade and in GABA-CB1R knockout mice}

We next evaluated the duration of the memory impairment associated with nicotine withdrawal by using the object-recognition task at several time points in different cohorts of mice (Figure 3A). A significant decrease in the discrimination index was observed at day $1(\mathrm{p}<0.01), 2(\mathrm{p}<0.01)$ and $4(\mathrm{p}<0.01)$ after the precipitation of withdrawal with mecamylamine. Mice recovered completely from the cognitive impairment 8 days after the precipitation of nicotine abstinence (Figure 3A). Subchronic treatment with rimonabant $(1 \mathrm{mg} / \mathrm{kg}$, once daily during 4 days $)$ prevented the memory impairment 4 days after withdrawal precipitation $(\mathrm{p}<0.05)$ (interaction treatment $\mathrm{x}$ pretreatment: $F_{1,39}=8.79, \mathrm{p}<0.01$ ) (Figure $3 \mathrm{~B}$ ), confirming the role played by $\mathrm{CB} 1 \mathrm{R}$ in this behavior. Total exploration time in the object-recognition task was not altered in these experiments (Figure S5). Given the involvement of protein synthesis in the cognitive deficits and the duration of this effect, we investigated whether possible changes in structural plasticity could underlie the memory impairment observed during nicotine withdrawal. Dendritic spine density and morphology were analyzed in key areas related to cognitive processes, such as the HPC and mPFC. Four days after the precipitation of nicotine withdrawal, when the cognitive deficits were still present, animals subchronically treated with vehicle or rimonabant $(1 \mathrm{mg} / \mathrm{kg})$ were sacrificed and 
their brains processed for ballistic delivery to label whole neurons with the dye DiI. Total dendritic spine density was not significantly altered in CA1 hippocampal pyramidal neurons (Figure 3C). Dendritic spines are classified into different groups depending on their morphology (stubby, mushroom, thin, branched and filopodia) and can undergo remodelling, that modifies their functionality (36). A decrease in the density of mushroom (mature) spines (interaction treatment $x$ pretreatment: $F_{1,26}=7.54$, $\mathrm{p}<0.01)$ was observed in nicotine abstinent mice $(\mathrm{p}<0.01)$ (Figure 3D). Notably, this reduced density of mature spines was normalized after a subchronic treatment with rimonabant $(\mathrm{p}<0.01)$, suggesting that this spine morphology alteration is regulated through CB1R (Figures 3D, E). Dendritic spine density and morphology of pyramidal mPFC neurons was not altered in nicotine abstinent mice (Figure S6). As previously shown, CB1R specifically located in GABAergic neurons are involved in the memory impairment related to nicotine abstinence. We next evaluated whether the activation of $\mathrm{CB} 1 \mathrm{R}$ in GABAergic neurons also mediate the structural plasticity alterations observed in nicotine abstinent mice. As expected, there was no difference in total spine density in saline or nicotine treated animals after the precipitation of withdrawal in CA1 hippocampal pyramidal neurons (Figure 3F). In agreement with the pharmacological experiments, a decrease of mushroom spine density was found in nicotine withdrawn WT animals in CA1 pyramidal neurons $(\mathrm{p}<0.01)$ (interaction treatment $\mathrm{x}$ pretreatment: $\left.\mathrm{F}_{1,18}=4.47, \mathrm{p}<0.05\right)$. Interestingly, this low density of mature spines was completely reversed in GABA-CB1R KO mice $(\mathrm{p}<0.01$ ) (Figures 3G, H). AMPA and NMDA glutamate receptors are involved in the regulation of synaptic plasticity $(37,38)$. A decrease of GluR2 expression was observed in the HPC during nicotine abstinence while no differences were observed in the expression of GluR1, GluR3, NR2A and 
NR2B subunits. Glutamate receptor expression was not altered in the mPFC of nicotine withdrawn mice (Figure S7).

As a whole, these results indicate that nicotine withdrawal involves changes in the density of mature spines in HPC, which are associated with the presence of cognitive deficits. Indeed, a significant correlation between memory performance (discrimination index values) and mushroom spine density in control and nicotine withdrawn mice (Figure 3I) was observed. Moreover, these alterations are mediated through CB1R specifically located in GABAergic neurons.

\section{Different neurobiological mechanisms mediate somatic signs and cognitive deficits}

\section{during nicotine withdrawal}

As previously shown, 2-AG levels are increased after the precipitation of nicotine withdrawal contributing to the cognitive impairment associated with this syndrome. We next studied the consequences of the pharmacological modulation of 2-AG levels in the somatic signs of nicotine abstinence. Interestingly, the administration of the MAGL inhibitor JZL184 decreased the severity of withdrawal, as recently reported (25), while the DAGL inhibitor O7460 exacerbated these symptoms (Figure 4A). Thus, two-way ANOVA showed an interaction between treatment and pretreatment $\left(F_{3,50}=13.63\right.$, $\mathrm{p}<0.05)$. Subsequent post hoc analysis revealed a decrease of the global withdrawal score by JZL184 at the dose of $20 \mathrm{mg} / \mathrm{kg}(\mathrm{p}<0.05)$, and an enhancement of this score by O7460 $(12 \mathrm{mg} / \mathrm{kg})(\mathrm{p}<0.05)$. These results suggest that $2-\mathrm{AG}$ is released after nicotine withdrawal to attenuate somatic signs of withdrawal producing simultaneously cognitive deficits, which would persist during longer time than physical symptoms. Moreover, these data indicate that different neurobiological mechanisms would be involved in the appearance of physical signs and memory impairment during nicotine 
abstinence. Indeed, the mTOR inhibitor temsirolimus $(1 \mathrm{mg} / \mathrm{kg})$ and the mGluR5 antagonist MTEP $(10 \mathrm{mg} / \mathrm{kg}$ ) abolished cognitive deficits (Figure 2D,G), but did not modify the severity of the somatic signs of withdrawal (interaction: $\mathrm{F}_{1,25}=0.44$, NS, and $\mathrm{F}_{1,25}=3.16$, NS, respectively) (Figure $4 \mathrm{~B}, \mathrm{C}$ ). Individual signs of withdrawal in the different experiments are shown in Table S1. 


\section{Discussion}

Our data show a pivotal role for CB1Rs, specifically those located in GABAergic cells, in the memory impairment and structural dendritic morphology alterations associated with the nicotine withdrawal syndrome. Moreover, we reveal that 2-AG is involved in this effect since an increase of this endocannabinoid was observed after the precipitation of withdrawal and the inhibition of its biosynthesis prevented cognitive deficits of nicotine abstinence. These data suggest that CB1R could be targeted to normalize cognition during early abstinence, which could have a clear therapeutic interest given that cognitive impairment may predict smoking relapse (6).

Despite the negative consequences of smoking, only approximately 3-5\% of smokers who attempt to quit on their own remain abstinent at 6 months (39), and around 50-75\% of smokers relapse during the first week of a quit attempt (5). Cognitive deficits that appear during early nicotine withdrawal seem to be involved in smoking relapse $(6,40)$, and therefore drugs improving withdrawal-related cognitive deficits could represent a strategy of treatment or serve as adjunctive pharmacotherapy for smokers most likely to experience these problems (7).

The precipitation of withdrawal with mecamylamine in nicotine-dependent mice after the training phase decreased the discrimination index in the object-recognition task, revealing a deficit in memory consolidation. Similar cognitive deficits have been shown in rodents in other hippocampal-dependent tasks such as the spatial object-recognition and contextual fear conditioning during spontaneous $(41,42)$ and precipitated nicotine withdrawal (43). Chronic nicotine treatment did not alter memory performance in C57BL/6J mice as previously reported $(43,44)$, suggesting that the cognitive deficits observed were specific of the withdrawal period. Pharmacological or genetic deletion of CB1R prevented memory impairment induced by nicotine abstinence. Moreover, these 
cognitive deficits were abolished in conditional $\mathrm{KO}$ mice lacking $\mathrm{CB} 1 \mathrm{R}$ in forebrain GABAergic neurons, the neuronal population in which CB1Rs are more abundantly expressed in the HPC. In agreement, the amnesic-like effects of THC, the main psychoactive component of Cannabis sativa, were demonstrated to be mediated by CB1Rs located in these particular neurons by using the same paradigm (23).

We identified the 2-AG as the endocannabinoid responsible for the cognitive deficits associated with the nicotine withdrawal syndrome. An increase of 2-AG, but not AEA, was observed 10 minutes after the precipitation of withdrawal. Notably, the selective inhibitor of DAG lipases O7460 (22), which are the enzymes in charge of 2-AG biosynthesis, totally prevented memory deficits. In contrast, the administration of JZL184 which inhibits MAGL, the enzyme responsible for 2-AG degradation, did not modify this effect. Indeed, JZL184 induced a cognitive impairment by itself at $20 \mathrm{mg} / \mathrm{kg}$, consistent with the observation that 2-AG hydrolysis blockade impairs learning and memory performance (32). Taken together, these data indicate that 2-AG and activation of CB1Rs in GABAergic neurons are required for the appearance of nicotine withdrawal-induced memory impairment.

mTOR signaling regulates many integrated physiological functions of the nervous system including neuronal development, synaptic plasticity, memory storage, and cognition (45), and perturbation of this cascade appears to be a common pathophysiological feature of human neurological disorders including those related to cognitive alterations (34). In agreement, activation of mTOR signaling was revealed to be necessary for the amnesic-like effects of THC (23). Interestingly, the administration of the mTOR inhibitor temsirolimus (46), as well as the protein synthesis inhibitor anysomicin, abolished the cognitive deficits present during nicotine abstinence. Thus, mTOR activation and excessive protein synthesis could also underlie the behavioral 
deficit induced by nicotine withdrawal. In this sense, studies using animal models have revealed an aberrant increase in protein synthesis in other conditions characterized by memory impairment such as the fragile $\mathrm{X}$ syndrome (35) or the administration of THC (23).

We next investigated the duration of the cognitive deficits associated with nicotine withdrawal and the possible existence of changes in structural plasticity during this syndrome. Memory impairment was still present 4 days after the precipitation of withdrawal and mice recovered from this deficit by the $8^{\text {th }}$ day of withdrawal. In agreement, a previous study showed a similar duration of these cognitive deficits in the hippocampal-dependent contextual fear conditioning test in C57BL/6J mice (47). Interestingly, morphological analysis of dendritic spines in the CA1 region of the HPC revealed a decrease of mushroom (matures) spines 4 days after the precipitation of nicotine withdrawal, when cognitive impairment was still observed. Accordingly, the number and shape of synapse-bearing spines in the hippocampal formation are dynamic $(48,49)$ and are regulated by several factors including stress $(50)$. The stressful condition of nicotine withdrawal could be responsible for the reduced density of mature spines, probably leading to synaptic dysfunction and cognitive deficits. However, the neurobiological mechanisms described in our study should not be generalized to other types of stressful conditions such as acute corticosterone injection. Thus, the administration of compounds affecting the endocannabinoid system such as MTEP and O7460 before an acute amnesic dose of corticosterone did not prevent memory impairment in the object-recognition test (Figure S8). On the other hand, the decrease of GluR2 expression observed in the HPC of nicotine withdrawn mice could be related to the reduced number of mature spines. Thus, AMPA glutamate receptors including GluR2 subunits (51) modulate synaptic strength, and are abundant in mushroom spines, 
but sparsely distributed in thin and filopodia spines of CA1 hippocampal pyramidal neurons (52). In line with our study in mice, cortical neuroplasticity in cigarette smokers was also altered during early withdrawal as revealed by using transcranial direct current stimulation (53). This effect on neuroplasticity might be relevant for the high probability of relapse in heavy smokers (53). However, under our experimental conditions, no modifications of structural plasticity were observed in pyramidal neurons of mPFC.

The reduction of mushroom-type spines in CA1 hippocampal neurons associated with nicotine withdrawal was normalized by a subchronic treatment with a low dose of rimonabant and in GABA-CB1R KO mice. Accordingly, the same CB1R antagonist reversed cognitive and hippocampal dendritic spine deficits in a model of fragile $\mathrm{X}$ syndrome (54). We did not observe modifications in the average spine density in CA1 pyramidal neurons in GABA-CB1R KO mice under basal conditions, although a reduction of this density was shown by a recent study (55). Different methodology used (apical versus apical and basal dendrites analysis) could explain this discrepancy. Taken together, these results suggest that $\mathrm{CB} 1 \mathrm{R}$ activation in GABAergic neurons induces modifications in the morphology of hippocampal dendritic spines leading to the appearance of cognitive deficits during early nicotine withdrawal. An important role for this population of $\mathrm{CB} 1 \mathrm{Rs}$ has been recently shown in other behavioral responses such as voluntary exercise performance (56), food intake (21), cocaine addiction (57), or anxiety (29).

2-AG seems to play divergent functional effects during nicotine abstinence. Thus, the pharmacological modulation of 2-AG levels induced opposite effects on the somatic signs and memory impairment revealed during withdrawal. The MAGL inhibitor JZL184 reduced the severity of nicotine physical dependence, as recently reported (25), 
while the inhibition of DAG lipases by O7460 (22) exacerbated somatic signs of withdrawal. These data suggest that 2-AG might be released during nicotine withdrawal to alleviate physical signs producing simultaneously cognitive deficits, which seem more persistent in time, and may be therefore more relevant for early relapse than physical symptoms. Several reports suggest that stress exposure increases 2-AG levels to counteract many of the negative effects of this response (58). Different neurobiological mechanisms seem to mediate these two aspects of nicotine abstinence. Thus, the mTOR inhibitor temsirolimus and the inhibitor of mGluR5 MTEP blocked memory impairment without affecting somatic signs. In agreement, no changes in the physical severity of nicotine abstinence were observed in a previous study using the mGluR5 antagonist MPEP (59). Although the administration of MTEP should reduce 2AG levels after nicotine withdrawal and therefore worsen somatic signs, other biochemical processes could compensate or mask the effect of MTEP in this response. Indeed, mGluR5 blockade is a very general mechanism that could lead to these compensatory mechanisms beyond the modulation of the endocannabinoid system. O7460 increased nicotine physical severity probably because this compound directly inhibits the enzyme in charge of 2-AG synthesis.

All together, our work reveals the crucial involvement of CB1Rs located in GABAergic cells in the cognitive impairment and neuronal plasticity changes in the HPC occurring during nicotine withdrawal. This subpopulation of CB1Rs could be targeted to prevent smoking relapse by increasing cognitive performance during early nicotine withdrawal syndrome. 


\section{Acknowledgments}

This work was supported by the "Ministerio de Economía y Competitividad-MINECO" (\#SAF2014-59648-P) to R.M and (BFU2012-33500 and BFU2015-68568-P) to A.O., the "Instituto de Salud Carlos III" (\#PI13/00042) to F.B. and (RETICS-RTA, \#RD12/0028/0023) to R.M., the "Generalitat de Catalunya-AGAUR" (\#2014-SGR1547) to R.M., "Plan Nacional sobre Drogas" (\#2014I019) to F.B., the European Commission (NeuroPain, \#HEALTH-F2-2013-602891) to R.M., FP7-PEOPLE-2013IEF-623638 and French State/Agence Nationale de la Recherche/IdEx (ANR-10-IDEX03-02) to A.B-G. We thank Alba Domènech, Victòria Valls-Comamala, Júlia Sala and Marc Ten for excellent technical assistance. Rocío Saravia is a recipient of a predoctoral fellowship from the Instituto de Salud Carlos III.

\section{Financial Disclosures}

The authors have no conflicts of interest to declare. 


\section{References}

1. Gowing LR, Ali RL, Allsop S, Marsden J, Turf EE, West R, Witton J (2015): Global statistics on addictive behaviours: 2014 status report. Addiction 110:904-919.

2. Jackson KJ, Muldoon PP, De Biasi M, Damaj MI (2015): New mechanisms and perspectives in nicotine withdrawal. Neuropharmacology 96(Pt B):223-234.

3. Wesnes KA, Edgar CJ, Kezic I, Salih HM, de Boer P (2013): Effects of nicotine withdrawal on cognition in a clinical trial setting. Psychopharmacology (Berl) 229:133140.

4. Hall FS, Der-Avakian A, Gould TJ, Markou A, Shoaib M, Young JW (2015): Negative affective states and cognitive impairments in nicotine dependence. Neurosci Biobehav Rev 58:168-185.

5. Ashare RL, Falcone M, Lerman C (2014): Cognitive function during nicotine withdrawal: Implications for nicotine dependence treatment. Neuropharmacology $76(\mathrm{Pt}$ B):581-591.

6. Patterson F, Jepson C, Loughead J, Perkins K, Strasser AA, Siegel S, et al. (2010): Working memory deficits predict short-term smoking resumption following brief abstinence. Drug Alcohol Depend 106:61-64.

7. Ashare RL, Schmidt HD (2014): Optimizing treatments for nicotine dependence by increasing cognitive performance during withdrawal. Expert Opin Drug Discov 9:579594.

8. Patterson F, Jepson C, Strasser AA, Loughead J, Perkins KA, Gur RC, et al. (2009): Varenicline improves mood and cognition during smoking abstinence. Biol Psychiatry 65:144-149. 
9. Davis JA, Gould TJ (2009): Hippocampal nAChRs mediate nicotine withdrawalrelated learning deficits. Eur Neuropsychopharmacol 19:551-561.

10. Gould TJ, Leach PT (2014): Cellular, molecular, and genetic substrates underlying the impact of nicotine on learning. Neurobiol Learn Mem 107:108-132.

11. Maldonado R, Valverde O, Berrendero F (2006): Involvement of the endocannabinoid system in drug addiction. Trends Neurosci 29:225-232.

12. Morena M, Campolongo P (2014): The endocannabinoid system: an emotional buffer in the modulation of memory function. Neurobiol Learn Mem 112:30-43.

13. Maldonado R, Berrendero F (2010): Endogenous cannabinoid and opioid systems and their role in nicotine addiction. Curr Drug Targets 11:440-449.

14. Gamaleddin IH, Trigo JM, Gueye AB, Zvonok A, Makriyannis A, Goldberg SR, Le Foll B (2015): Role of the endogenous cannabinoid system in nicotine addiction: novel insights. Front Psychiatry 6:41

15. Cohen C, Perrault G, Voltz C, Steinberg R, Soubrié P (2002): SR141716, a central cannabinoid $(\mathrm{CB}(1))$ receptor antagonist, blocks the motivational and dopaminereleasing effects of nicotine in rats. Behav Pharmacol 13:451-463.

16. Navarrete F, Rodríguez-Arias M, Martín-García E, Navarro D, García-Gutiérrez MS, Aguilar MA, et al. (2013): Role of CB2 cannabinoid receptors in the rewarding, reinforcing, and physical effects of nicotine. Neuropsychopharmacology 38:2515-2524. 17. Gamaleddin I, Zvonok A, Makriyannis A, Goldberg SR, Le Foll B (2012): Effects of a selective cannabinoid CB2 agonist and antagonist on intravenous nicotine self administration and reinstatement of nicotine seeking. PLoS One 7:e29900

18. Forget B, Guranda M, Gamaleddin I, Goldberg SR, Le Foll B (2016): Attenuation of cue-induced reinstatement of nicotine seeking by URB597 through cannabinoid CB1 receptor in rats. Psychopharmacology (Berl) 233:1823-1828. 
19. Cahill K, Ussher M (2011): Cannabinoid type 1 receptor antagonists for smoking cessation. Cochrane Database Syst Rev (3):CD005353.

20. Monory K, Massa F, Egertová M, Eder M, Blaudzun H, Westenbroek R, et al. (2006): The endocannabinoid system controls key epileptogenic circuits in the hippocampus. Neuron 51:455-466.

21. Bellocchio L, Lafenêtre P, Cannich A, Cota D, Puente N, Grandes P, et al. (2010): Bimodal control of stimulated food intake by the endocannabinoid system. Nat Neurosci 13:281-283.

22. Bisogno T, Mahadevan A, Coccurello R, Chang JW, Allarà M, Chen Y, et al. (2013): A novel fluorophosphonate inhibitor of the biosynthesis of the endocannabinoid 2-arachidonoylglycerol with potential anti-obesity effects. $B r$ J Pharmacol 169:784793.

23. Puighermanal E, Marsicano G, Busquets-Garcia A, Lutz B, Maldonado R, Ozaita A (2009): Cannabinoid modulation of hippocampal long-term memory is mediated by mTOR signaling. Nat Neurosci 12:1152-1158.

24. Busquets-Garcia A, Puighermanal E, Pastor A, de la Torre R, Maldonado R, Ozaita A (2011): Differential role of anandamide and 2-arachidonoylglycerol in memory and anxiety-like responses. Biol Psychiatry 70:479-486.

25. Muldoon PP, Chen J, Harenza JL, Abdullah RA, Sim-Selley LJ, Cravatt BF, et al. (2015): Inhibition of monoacylglycerol lipase reduces nicotine withdrawal. $\mathrm{Br} J$ Pharmacol 172:869-882.

26. Ennaceur A (2010): One-trial object recognition in rats and mice: methodological and theoretical issues. Behav Brain Res 215:244-254. 
27. Jacob W, Yassouridis A, Marsicano G, Monory K, Lutz B, Wotjak CT (2009): Endocannabinoids render exploratory behaviour largely independent of the test aversiveness: role of glutamatergic transmission. Genes Brain Behav 8:685-698.

28. Häring M, Kaiser N, Monory K, Lutz B (2011): Circuit specific functions of cannabinoid $\mathrm{CB} 1$ receptor in the balance of investigatory drive and exploration. PLoS One 6(11):e26617.

29. Rey AA, Purrio M, Viveros MP, Lutz B (2012): Biphasic effects of cannabinoids in anxiety responses: $\mathrm{CB} 1$ and $\mathrm{GABA}(\mathrm{B})$ receptors in the balance of GABAergic and glutamatergic neurotransmission. Neuropsychopharmacology 37:2624-2634.

30. Di Marzo V (2011): Endocannabinoid signaling in the brain: biosynthetic mechanisms in the limelight. Nat Neurosci 14:9-15.

31. Di Marzo V, De Petrocellis L, Bisogno T (2005): The biosynthesis, fate and pharmacological properties of endocannabinoids. Handb Exp Pharmacol (168):147185.

32. Griebel G, Pichat P, Beeské S, Leroy T, Redon N, Jacquet A, et al. (2015): Selective blockade of the hydrolysis of the endocannabinoid 2-arachidonoylglycerol impairs learning and memory performance while producing antinociceptive activity in rodents. Sci Rep 5:7642.

33. Costa-Mattioli M, Monteggia LM (2013): mTOR complexes in neurodevelopmental and neuropsychiatric disorders. Nat Neurosci 16:1537-1543.

34. Hoeffer CA, Klann E (2010): mTOR signaling: at the crossroads of plasticity, memory and disease. Trends Neurosci 33:67-75.

35. Bolduc FV, Bell K, Cox H, Broadie KS, Tully T (2008): Excess protein synthesis in Drosophila fragile X mutants impairs long-term memory. Nat Neurosci 11:1143-1145. 
36. Bourne JN, Harris KM (2008): Balancing structure and function at hippocampal dendritic spines. Annu Rev Neurosci 31:47-67.

37. Huganir RL, Nicoll RA (2013): AMPARs and synaptic plasticity: the last 25 years. Neuron 80:704-717.

38. Paoletti P, Bellone C, Zhou Q (2013): NMDA receptor subunit diversity: impact on receptor properties, synaptic plasticity and disease. Nat Rev Neurosci 14:383-400.

39. Hughes JR, Keely J, Naud S (2004): Shape of the relapse curve and long-term abstinence among untreated smokers. Addiction 99:29-38.

40. Loughead J, Wileyto EP, Ruparel K, Falcone M, Hopson R, Gur R, Lerman C (2015): Working memory-related neural activity predicts future smoking relapse. Neuropsychopharmacology 40:1311-1320.

41. Kenney JW, Adoff MD, Wilkinson DS, Gould TJ (2011): The effects of acute, chronic, and withdrawal from chronic nicotine on novel and spatial object recognition in male C57BL/6J mice. Psychopharmacology (Berl) 217:353-365.

42. Wilkinson DS, Turner JR, Blendy JA, Gould TJ (2013): Genetic background influences the effects of withdrawal from chronic nicotine on learning and high-affinity nicotinic acetylcholine receptor binding in the dorsal and ventral hippocampus. Psychopharmacology (Berl) 225:201-208.

43. Raybuck JD, Gould TJ (2009): Nicotine withdrawal-induced deficits in trace fear conditioning in C57BL/6 mice--a role for high-affinity beta2 subunit-containing nicotinic acetylcholine receptors. Eur J Neurosci 29:377-387.

44. Davis JA, James JR, Siegel SJ, Gould TJ (2005): Withdrawal from chronic nicotine administration impairs contextual fear conditioning in C57BL/6 mice. J Neurosci 25:8708-8713. 
45. Bockaert J, Marin P (2015): mTOR in Brain Physiology and Pathologies. Physiol Rev 95:1157-1187.

46. Puighermanal E, Busquets-Garcia A, Gomis-González M, Marsicano G, Maldonado R, Ozaita A (2013): Dissociation of the pharmacological effects of THC by mTOR blockade. Neuropsychopharmacology 38:1334-1343.

47. Gould TJ, Portugal GS, André JM, Tadman MP, Marks MJ, Kenney JW, et al. (2012): The duration of nicotine withdrawal-associated deficits in contextual fear conditioning parallels changes in hippocampal high affinity nicotinic acetylcholine receptor upregulation. Neuropharmacology 62:2118-2125.

48. Bourne JN, Harris KM (2008): Balancing structure and function at hippocampal dendritic spines. Annu Rev Neurosci 31:47-67.

49. Kasai H, Fukuda M, Watanabe S, Hayashi-Takagi A, Noguchi J (2010): Structural dynamics of dendritic spines in memory and cognition. Trends Neurosci 33:121-129.

50. Maras PM, Baram TZ (2012): Sculpting the hippocampus from within: stress, spines, and CRH. Trends Neurosci 35:315-324.

51. Chater TE, Goda Y (2014): The role of AMPA receptors in postsynaptic mechanisms of synaptic plasticity. Front Cell Neurosci 8:401.

52. Matsuzaki M, Ellis-Davies GC, Nemoto T, Miyashita Y, Iino M, Kasai H (2001): Dendritic spine geometry is critical for AMPA receptor expression in hippocampal CA1 pyramidal neurons. Nat Neurosci 4:1086-1092.

53. Grundey J, Thirugnanasambandam N, Kaminsky K, Drees A, Skwirba AC, Lang N, et al. (2012): Neuroplasticity in cigarette smokers is altered under withdrawal and partially restituted by nicotine exposition. J Neurosci 32:4156-4162. 
54. Busquets-Garcia A, Gomis-González M, Guegan T, Agustín-Pavón C, Pastor A, Mato S, et al. (2013): Targeting the endocannabinoid system in the treatment of fragile X syndrome. Nat Med 19:603-607.

55. Monory K, Polack M, Remus A, Lutz B, Korte M (2015): Cannabinoid CB1 receptor calibrates excitatory synaptic balance in the mouse hippocampus. J Neurosci $35: 3842-3850$.

56. Dubreucq S, Durand A, Matias I, Bénard G, Richard E, Soria-Gomez E, et al. (2013): Ventral tegmental area cannabinoid type-1 receptors control voluntary exercise performance. Biol Psychiatry 73:895-903.

57. Martín-García E, Bourgoin L, Cathala A, Kasanetz F, Mondesir M, GutiérrezRodriguez A, et al. (2015): Differential Control of Cocaine Self-Administration by GABAergic and Glutamatergic CB1 Cannabinoid Receptors. Neuropsychopharmacology 2015 Nov 27. doi: 10.1038/npp.2015.351

58. Morena M, Patel S, Bains JS, Hill MN (2016): Neurobiological Interactions Between Stress and the Endocannabinoid System. Neuropsychopharmacology 41:80102

59. Liechti ME, Markou A (2007): Interactive effects of the mGlu5 receptor antagonist MPEP and the mGlu2/3 receptor antagonist LY341495 on nicotine self-administration and reward deficits associated with nicotine withdrawal in rats. Eur J Pharmacol 554:164-174. 


\section{Figure legends}

\section{Figure 1}

CB1R mediates the cognitive deficits associated with nicotine withdrawal. (A) Nicotine withdrawal was precipitated with mecamylamine $(2 \mathrm{mg} / \mathrm{kg}) 20$ minutes after the training session, and discrimination index was obtained 24 hours after the training $(\mathrm{n}=11$ mice per group). (B) Discrimination index obtained after chronic treatment with saline or nicotine during 13 days ( $\mathrm{n}=6-7$ mice per group). (C) Rimonabant $(1 \mathrm{mg} / \mathrm{kg}$ ) was administered immediately after the training session 20 minutes before the precipitation of nicotine withdrawal, and discrimination index was obtained 24 hours after the training ( $\mathrm{n}=5-7$ mice per group). (D-E) Nicotine withdrawal was precipitated with mecamylamine $(2 \mathrm{mg} / \mathrm{kg}) 20$ minutes after the training session in (D) WT and CB1R KO mice ( $=$ 5-6 mice per group), and in (E) WT and GABA-CB1R KO, Glu-CB1R KO and GABA/Glu CB1R KO mice ( $\mathrm{n}=5-8$ mice per group), and discrimination index was obtained 24 hours after the training. Data are expressed as mean \pm SEM. $\star \star p<0.01$ (compared with saline), \#p<0.05 (comparison between genotypes) \#\#p<0.01 (comparison between pretreatments or genotype). WT, wild-type mice; $\mathrm{KO}$, knockout mice.

\section{Figure 2}

2-arachidonoylglycerol is involved in the cognitive deficits associated with nicotine withdrawal. (A) Schematic representation of the experimental design for (B,C) endocannabinoids measure. Levels of (B) 2-arachidonoylglycerol and (C) anandamide in whole brain homogenates extracted 10 minutes after the precipitation of nicotine withdrawal, measured by liquid chromatography-mass spectrometry $(n=8$ mice per group). (D) Protein levels of DAGL- $\alpha$ and MAGL in whole brain homogenates 10 
minutes after the precipitation of nicotine withdrawal ( $n=6$ mice per group). (E,F,G,H,J) MTEP (10mg/kg), O7460 (12mg/kg), temsirolimus (1mg/kg), and anysomicin $(6 \mathrm{mg} / \mathrm{kg})$ were administered immediately after the training session 20 minutes before the precipitation of nicotine withdrawal. JZL184 (8 and 20mg/kg) was administered immediately after the training session 2 hours before the precipitation of nicotine withdrawal. Discrimination index was obtained 24 hours after the training $(\mathrm{n}=$ 7-11 mice per group). (I) Anysomicin (4, 6, 8, 12 and 18mg/kg) was administered immediately after the training session, and discrimination index was obtained 24 hours after the training $(\mathrm{n}=5-12$ mice per group). Data are expressed as mean \pm SEM. $\star p<0.05$ (compared with vehicle); $\star \star p<0.01$ (compared with saline), ${ }^{\# \#} p<0.01$ (comparison between pretreatments).

\section{Figure 3}

Structural plasticity changes associated with nicotine withdrawal are normalized by CB1R blockade and in GABA-CB1R knockout mice. (A) Schematic representation of the experimental design used to evaluate the duration of the cognitive deficits associated with nicotine abstinence. Nicotine withdrawal was precipitated with mecamylamine $(2 \mathrm{mg} / \mathrm{kg}) 20$ minutes after the training session, and discrimination index was obtained 1 , 2, 4 and 8 days after the training in different cohorts of mice $(n=9-12$ mice per group). (B) Schematic representation of the experimental design used to evaluate the effects of subchronic treatment with rimonabant in the cognitive deficits observed 4 days after the precipitation of nicotine withdrawal. Rimonabant was injected at $1 \mathrm{mg} / \mathrm{kg}$, once daily during 4 days ( $\mathrm{n}=9-12$ mice per group). (C) Overall dendritic spine density, (D) Analysis of spine morphology and (E) Representative DiOlistics staining of CA1 hippocampal pyramidal neurons of mice subcronically treated with vehicle or 
rimonabant $(1 \mathrm{mg} / \mathrm{kg}$, once daily during 4 days $)$ evaluated 4 days after the precipitation of nicotine withdrawal ( $\mathrm{n}=7-8$ mice per group). (F) Overall dendritic spine density, (G) Analysis of spine morphology and (H) Representative DiOlistics staining of CA1 hippocampal pyramidal neurons of WT and GABA-CB1R knockout mice evaluated 4 days after the precipitation of nicotine withdrawal ( $n=4-7$ mice per group). Scale bar $=$

$2 \mu \mathrm{m}$. Arrows indicate mushroom (mature) spines. (I) Correlation between memory performance (discrimination index values) and mushroom spine density 4 days after the precipitation of nicotine withdrawal. Data are expressed as mean \pm SEM. $\star \star p<0.01$ (compared with saline), ${ }^{\#} p<0.05$ (comparison between pretreatments), ${ }^{\# \#} p<0.01$ (comparison between pretreatments or genotypes). WT, wild-type mice; KO, knockout mice.

\section{Figure 4}

Different neurobiological mechanisms underlie cognitive deficits and somatic signs of nicotine withdrawal. Mecamylamine $(2 \mathrm{mg} / \mathrm{kg})$ was administered to precipitate withdrawal in nicotine dependent mice. O7460 (12mg/kg), temsirolimus $(1 \mathrm{mg} / \mathrm{kg})$ and MTEP (10mg/kg) were injected 20 minutes before mecamylamine. JZL184 (8 and $20 \mathrm{mg} / \mathrm{kg}$ ) was injected 2 hours before mecamylamine. A global withdrawal score was calculated by giving each individual sign a relative weight in (A) vehicle, JZL184 and O7460 pretreated mice ( $n=6-10$ mice per group), in $(\mathbf{B})$ temsirolimus pretreated mice ( $n=7-8$ mice per group), and in (C) MTEP pretreated mice ( $n=7-8$ mice per group).

Data are expressed as mean \pm SEM. $\star \star p<0.01$ (compared with saline), ${ }^{*} p<0.05$ (comparison between pretreatments). 